\title{
Developments in Segmentation of Abdominal Liver CT Images for Cancer and Other Disease Lesion Detection
}

\author{
Hariharan $S^{*}$ \\ Professor in Electrical Engineering, College of Engineering Trivandrum, India \\ *Corresponding author: Hariharan S, Professor in Electrical Engineering, College of \\ Engineering Trivandrum, India, Email: harikerala2001@yahoo.com
}

\section{Research Article \\ Volume 2 Issue 1}

Received Date: December 29, 2018

Published Date: February 22, 2019

DOI: $10.23880 /$ aabsc-16000114

\begin{abstract}
Segmentation is one of the most important and widely used methods in medical image analysis. It is considered to be a high level image processing technique and can be used for many applications in medical imaging. CT images are commonly used in medical field and it provides clear picture of the internal organs. However in some places further processing of CT images are required for disease diagnosis and lesion detection. This work is an effort for bringing out clinical information from liver images of computed tomography based on image processing. Finally liver tumor classifications have been performed using texture based image analysis.
\end{abstract}

Keywords: Medical imaging; Image Segmentation; Image Analysis; CT Liver Image Analysis

\section{Introduction}

Segmentation techniques are useful in partitioning regions of interest in an image. We can also separate different regions in an image by means of segmentation. There are two different ways for segmenting an image. The first one is the edge based segmentation and the other one is the region based segmentation. Edge based segmentation mainly make use of gradient operators such as Sobel, Canny, Prewitt, Compas, Roberts, Gaussion etc. It also includes various types of filtering techniques. Classical image segmentation techniques include region growing, splitting and merging thresholding etc. There are several modifications of the above methods which are available in the literature of segmentation research [1-10]. Segmenting the lesion of tumors or cysts from a CT liver image is not very easy because of the following reasons. Liver is a hidden organ which is difficult for observing.
Finding the lesion where tumor or cyst is visible is another difficult task. However, with the use of fine turned segmentation techniques, it is possible to extract the region of interest in an image. One approach for segmenting liver is by the use of classical segmentation techniques. Other techniques which are possible are by the use of modified segmentation techniques. One of the most simple image segmentation techniques is thresholding. There are various modifications of this method which can be classified as hard and soft thresholding techniques and are well implemented in the literature [11-20].

Filtering is one of the most important but simple operation in image analysis. In this method the noise contained in the image is filtered out by means of some filters such as wavelet filter and fuzzy filters. There are two types of noise commonly percent in images such as 
luminance noise and chroma noise. Luminance noise makes an image grainy but it is not visible when printed. Chroma noise can be visualized as red and blue pixels. If we remove the luminous noise it reduces the sharpness of the image. Removing the chroma noise will damage its actual colour. Reducing noise is actually equilibrium between softness and color damage.

Some of the latest trends in CT image analysis are by making use of wavelet based filtering [20-25]. The quality of the image can be improved by filtering by means of wavelet based filters and fuzzy filters [26-30]. Another verity of algorithms available is by using level set methods [31-35]. In this approach energy minimization is found to be one of the most important criterions. Researchers have developed several image analysis methods based on thresholding in hard and soft platforms [36-40].

In general edge based segmentation can be made possible by means of gradient operators and level set based approaches. Edge detection with gradient operators is not new, but it is used as a powerful tool in many areas of image processing and pattern recognition. They are found to be one of the most useful methods for detecting object edges. After detecting the object edges it is possible to find the area, volume etc of the tumor / cysts or other lesions present in the abdominal CT image.

In the present work, a brief review of the latest developments that took place in the field of segmentation research is described. Several methods are now available for segmenting an image based on the requirements of each and every one. There are few standard techniques available with image processing applications. Numerous modified techniques have been developed and the process is continuing day by day based on the requirements and the field of applications. This work is concentrating only in the field of liver image analysis. Large volume of literature is available now in liver image analysis and each one is having its own merits and demerits. In this work we tried to implement only simple methods which are easy to implement and having less computational complexity. The methods used here are gradient operators, various filtering techniques; level set methods, hard and soft thresholding etc.

\section{Materials and Methods}

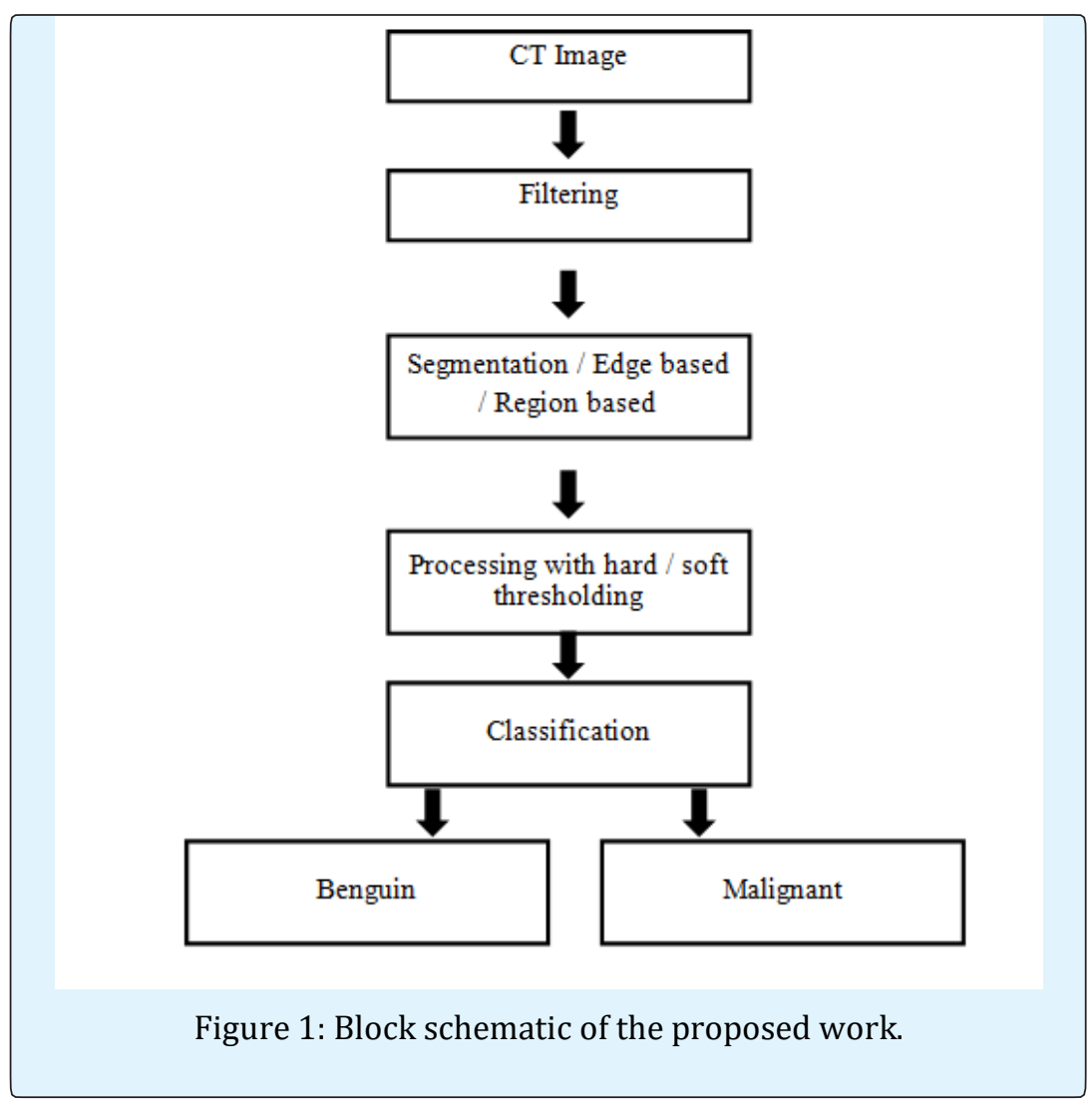

Hariharan S. Developments in Segmentation of Abdominal Liver CT Images for Cancer and Other Disease Lesion Detection. Ann Adv Biomed Sci 2019, 2(1): 000113. 
- In this work, different image processing operations are performed on abdominal liver CT images. The aim is to examine whether any clinical information can be dawn for disease diagnosis or therapy.

- Cancer detection and prevention is one of the most important and essential work and hence several medical professionals and biomedical engineers are involved in it. CT images are quite clear and to some extent human visual system is satisfied to provide diagnostic clues. Without disturbing the human body system, we can have a detailed study using imaging techniques. But some sort of subjectivity exists while examining these medical images. This will cause difficulty in decision making. Radiologists and medical professional requires more information in the above cases. Hence further processing of these images is required, in many cases.

- In the present work image data base have been prepared among various types of liver cancer images, collected from regional cancer center and medical colleges in and around Trivandrum, Kerala, India for conducting the study. 600 images of liver tumors, Cysts, Cirrhosis etc. have been collected. The images are resized into $256 \times 256$ pixels. Most of the images we got are in dicom format. These have been converted into GIF Format for easy processing. Various image processing operations are performed in these images and the results are shown to the radiologists and medical professionals. The selected images alone are used here for formulating the result and discussion parts.

\section{Support Vector Machine for Classification}

Support vector points will be lying on the margin. The exact solution to the classification problem can be expressed by the decision function

$$
d(x)=\operatorname{sign}\left[\sum_{i=1}^{N} A_{i} Y_{i} n\left(s_{i}, x\right)+b\right]
$$

Where

$A_{i}=$ Langrange multiplier; $x_{i} \in \mathcal{R}^{n}$ ( $i^{\text {th }}$ input vector), $y_{i} \in\{+1,-1\}$

$\mathrm{Si}=$ Support Vectors

$\mathrm{N}=$ Total number of support vectors

$n\left(s_{i}, x\right)=$ The function for convolution of Kernels

Radial Kernels usually provides the best result

$$
n(x, y)=e^{\left(-n(x-y)^{2}\right)}
$$

When output of sub image is 1 the system classifies the image as malignant. When it is -1then it classifies as benign.

Out of the 6oo liver tumor images 100 malignant liver tumors and the same number of benign tumor images are separated. Texture analysis has been performed on these images and texture features are extracted. These features are then used for classification of liver tumors into benign and malignant tumors. For the purpose of classification support vector machine is employed. Classified output is compared with the result that obtained by radiologists and medical experts.

\section{Methods of Approach}

\section{Gradient Operators}

Edge detection with gradient operators is found to be very effective in classical image processing processes. They are found to be one of the simplest methods by simply taking the first derivative of the image for detecting object edges. Many gradient operators such as Canny, Sobel, Prewitt, Robert, Compass etc. are available for finding the object edges. After detecting the object edges it is possible to find the area, volume etc of the tumors or lesions present in the abdominal CT images of liver. Even though edge detection is considered as a simple basic operation in image processing over the years researchers have identified the significance of detected edges. There are several advantages and disadvantages of these edge detection methods. Several methods contribute information losses from the original image. Some methods make the object edges thick. This further requires another step for edge thinning operation. After detection of the object edges it is possible to visualize the cyst or tumor lesion present in the abdominal CT image.

\section{Filtering}

Another simple operation which can be implemented in images of CT are filtering. We have several Filters such as linear filters and nonlinear filters which can be applied on quality image which can be used for further processing. Researches and medical professionals are always interested in reading the line good quality images. High pass, low pass etc., are some of the examples of commonly used filters. In image processing and pattern recognition we use special type of filter such as wavelet filters, fuzzy filters, various variations of median filters etc. which are capable of providing very good quality input images for further processing. Using filter banks is another idea which can provide excellent results.

$\eta \in \mathrm{R}$ and is a non - zero parameter

The SVM output is -1 or +1 


\section{Level Set Based Approach}

Computed tomography images can be used for liver tumor diagnosis. Accurate segmentation is essential for medical image analysis. Active contour methods can be used for the segmentation of tumors in liver CT images. Active contour methods have many disadvantages also. We will not get proper segmentation in the presence of noise and other major edges near to the original boundary. This can easily be carried out using an expectation maximization algorithm.

Level set methods can be used for modeling the liver tumor using different methods such as Mumford Shah model, Baysian level set model etc. However they are found to be very old. The piecewise constant model is one alternate solution to Mumford Shah model. This is first proposed by chan and vese. This method can be easily implemented on liver CT images and hence used for tumor segmentation.

\section{Hard Thresholding}

Image segmentation is one of the most important processes in image analysis. In segmentation we can partition an image into several sub regions. Out of these sub regions we can perform further analysis on the region of interest (RIO). There are many ways in which segmentation can be executed. The methods include region growing, splitting and merging, thresholding etc. Several algorithms have been proposed by segmentation researchers based on the above classical segmentation methods.

Thresholding is one of the most important and popular tool used for image segmentation. In thresholding usually two thresholds are fixed. One for the object and other for the back ground. Thresholding can be divided into global thresholding and local thresholding. In global thresholding we select a threshold ' $\mathrm{T}$ ' that will separate object from background. In local thresholding we divide an image into sub regions and perform thresholding in each region. Global thresholding technique is one that thresholds the entire image with a single threshold value. Local thresholding technique is one that partitions a given image into sub images and determines a threshold for each of these sub images. One of the best methods of thresholding is adaptive thresholding. In adaptive thresholding each pixel in an image is thresholded based on the histogram of the pixel neighborhood. Many researchers developed hard thresholding algorithms slightly varying from the original method. Out of which the methods developed by Otsu, Kapur, Sahoo PK, etc. are of considerable interest [36-38].

\section{Soft Thresholding Based Approach}

\section{Fuzzy Based Thresholding}

Fuzzy set theory proposed by Zadeh LA is one of the most important work for the analysis of medical images. Due to its flexibility, imprecise and natural characteristics this can very easily be implemented on medical image analysis similar to other classical segmentation algorithms. Fuzzy based approaches can be implemented on liver tumor segmentation process. However one with the multi-level thresholding provides fine turned segmented output. In most simple form, in this work fuzzy thresholding based strategy is used for the segmentation of tumors in liver CT images of the abdomen. Using this method very good segmentation can be achieved. This thresholding can be done in the crisp form or non-fuzzy platform or in the fuzzy platform. The fuzzy based approaches are found to be most suitable for medical images such as liver images. Hence in this work fuzzy thresholding is used for the segmentation of liver CT images.

\section{Wavelet Based Thresholding}

Wavelet based thresholding is another option. It is a powerful tool for noise reduction in an image because of its capability to bring out detailed spatial frequency information in real time. Due to this property it is possible to distinguish between noise and the original data. If we take wavelet transform of a data we get one representation which contain main structure of the image with some large efficient and the noise with the remaining small coefficients. Noise can be represented as a normal distribution or Gaussian distribution. Image denoising can be done with wavelet thresholding or shrinkage schemes like sure shrink or Bayes Shrink etc. This can be performed by thresholding small wavelet coefficients. If we threshold an image with global threshold and filter out small coefficients to remove noise, high frequency components belong to the edges will also remove which will result in image blurring. For improving the wavelet based denoising local variance evaluation can be done. Local variance can characterize local features of an image. Area with small variation provides homogenous region where as regions containing edges will have a higher variance. In this work wavelet based thresholding is used for reducing noise and preserve the important edges in the image [46-50]. 


\section{Texture Based Approach}

Texture analysis is one of the most interesting areas in digital image processing mainly for image analysis and classification. It is commonly used for interpretation and classification of different types of images. In order to discriminate images with different texture characteristics it is necessary to extract texture features which embody information about the spatial distribution of grey level variations in each image. These texture features can be used directly from the image or from frequency domain. Based on the application they can be used for solving classification problems. In this work neighboring grey level depended matrix method is used for classification of liver tumor images.

\section{Results and Discussions}

First of all NGLDM feature are extracted from the original images in the data base. The mean and standard deviation are calculated for different texture features and or benign and malignant cases. It is shown in table 1. By trial and error it is observed that the features long run emphasis and entropy provided best classification results.

Cross validation method is used here for estimating the performance of the present system. Images in the data base are divided into $\mathrm{n}$ classes. The starting group is kept as such and the balance (n-1) group is used for training the SVM.

Figure 5 shows the performance of the SVM system with varying eta values. Between the bandwidth of 0.02 to 0.03 the proposed system provided stable and highest accuracy. SVM is tested on the group that was said aside. The second group is then removed, and the remaining n-1 group is trained and the network is tested on the excluded group. The method is continued until all $\mathrm{n}$ groups have been used. In this work the value of $\mathrm{n}$ is taken as 10 .

Neighboring grey level depended matrix method is briefly explained below.

Let the image be

$\begin{array}{lllll}1 & 1 & 2 & 3 & 1 \\ 0 & 1 & 1 & 2 & 2 \\ 0 & 0 & 2 & 2 & 1 \\ 3 & 3 & 2 & 2 & 1 \\ 0 & 0 & 2 & 0 & 1\end{array}$

The $\mathrm{P}$ matrix computed from the image is given below.

\begin{tabular}{l|lllllllll}
0 & 1 & 2 & 3 & 4 & 5 & 6 & 78
\end{tabular}

\begin{tabular}{l|llllllllll}
0 & 0 & 0 & 1 & 0 & 0 & 0 & 0 & 0 & 0
\end{tabular}

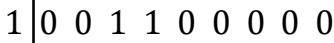

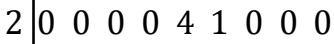

$30 \begin{array}{lllllllllll}0 & 1 & 0 & 0 & 0 & 0 & 0 & 0 & 0\end{array}$

The texture features computed from this method are directionally invariant. These features are first proposed by Sun and Wee [52]. The texture features proposed by them are small number emphasis, large number emphasis, second moment, number non uniformity and entropy. The method makes use of one element and its entire neighboring element at once instead of one direction at a time. This will reduce the calculation required in processing an image. The matrix $\mathrm{P}(\mathrm{i}, \mathrm{j})$ is a frequency count of grey level variations. $\mathrm{Ng}$ is the number of grey levels present in the image and $\mathrm{Nr}$ is the number of possible neighbors to a pixel in an image. In the above image P matrix is computed for grey levels $0-3$ at $d=1$ and $a=0$. For conducting the experiments these features are first extracted and then used for classification purpose.

\begin{tabular}{|c|c|c|c|c|}
\hline & \multicolumn{2}{|c|}{ Malignant } & \multicolumn{2}{c|}{ Benign } \\
\hline Sl no & Mean & Std. deviation & Mean & Std. deviation \\
\hline 1 & 0.9082 & 0.0184 & 0.906 & 0.0181 \\
\hline 2 & 0.9189 & 0.0163 & 0.922 & 0.0161 \\
\hline 3 & 0.8851 & 0.0236 & 0.8526 & 0.025 \\
\hline 4 & 0.8676 & 0.304 & 0.8676 & 0.027 \\
\hline 5 & 0.8556 & 0.299 & 0.8588 & 0.03 \\
\hline 6 & 0.8044 & 0.777 & 0.8132 & 0.0346 \\
\hline 7 & 0.8081 & 0.781 & 0.818 & 0.0324 \\
\hline 8 & 0.8016 & 0.7633 & 0.78 & 0.0394 \\
\hline 9 & 0.8008 & 0.75 & 0.771 & 0.0436 \\
\hline 10 & 0.7766 & 0.755 & 0.6616 & 0.048 \\
\hline
\end{tabular}

Table 1: Mean and standard deviations of the texture feature large number emphasis. 


\begin{tabular}{|c|c|c|}
\hline Test set & Malignant & Benign \\
\hline 1 & $2 / 10$ & $0 / 10$ \\
\hline 2 & $6 / 10$ & $0 / 10$ \\
\hline 3 & $1 / 10$ & $0 / 10$ \\
\hline 4 & $3 / 10$ & $0 / 10$ \\
\hline 5 & $5 / 10$ & $3 / 10$ \\
\hline 6 & $1 / 10$ & $3 / 10$ \\
\hline 7 & $1 / 10$ & $1 / 10$ \\
\hline 8 & $1 / 10$ & $1 / 10$ \\
\hline 9 & $1 / 10$ & $1 / 10$ \\
\hline 10 & $0 / 10$ & $0 / 10$ \\
\hline
\end{tabular}

Table 2: Misdiagnosed cases.

Gradient operators are set of kernels which are useful for edge detection. We have applied all the important edge detectors like Soble, Canny, Prewitt, Compass,
Gaussian etc. to get any clinical information present in the original image. However after performing all these operations on CT images of liver cancer and easily distinguishable features could not be brought out except very few methods such as sobel and canny. Other gradient operators performed lesser than the above two methods in bringing the clinical information from the input images. Like other studies we have also observed that accurate edge detection is not possible with them and smooth edge. Also it enhances the unwanted edges also which makes the output confusing. However it is easy to implement and detects edges and their orientation. The method is time consuming and difficult to implement. On the other hand canny edge detector having a smoothing effect for removing noise. This operator has got good localization and response. Also it enhances $\mathrm{S} / \mathrm{N}$ ratio and is immune to noisy environment.

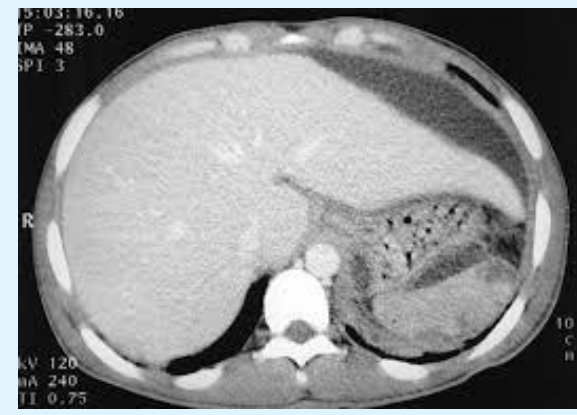

Figure 2(a): Original image.Figure
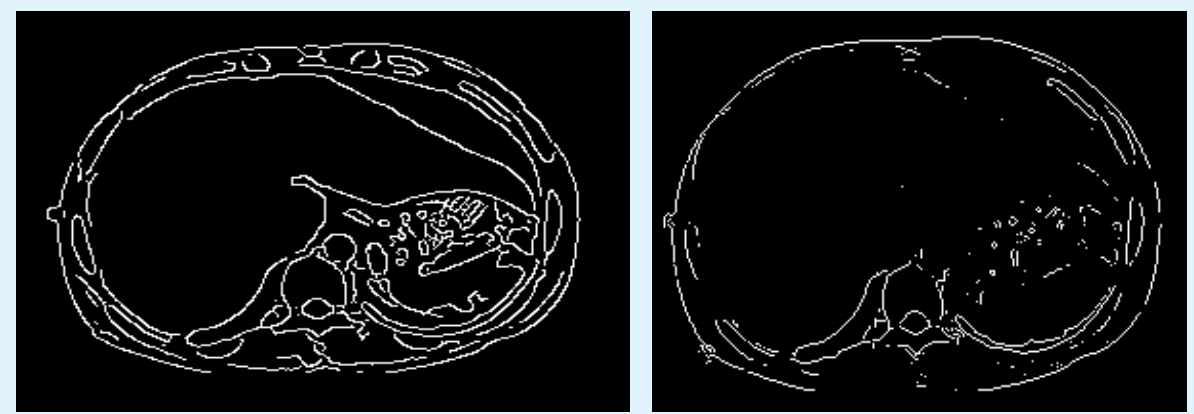

2(b): output of canny edge detector. Figure 2 (c): Output of Sobel edge detector.

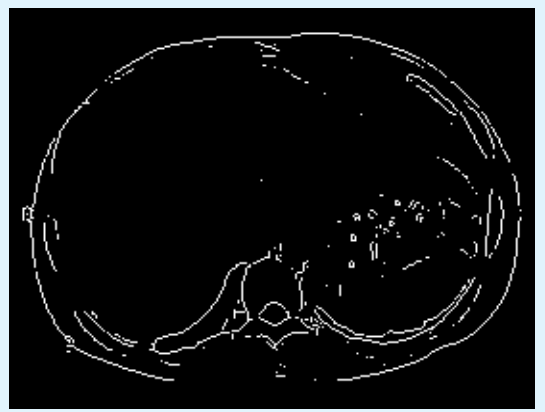

Figure 2(d): Output of Prewitt edge detector.

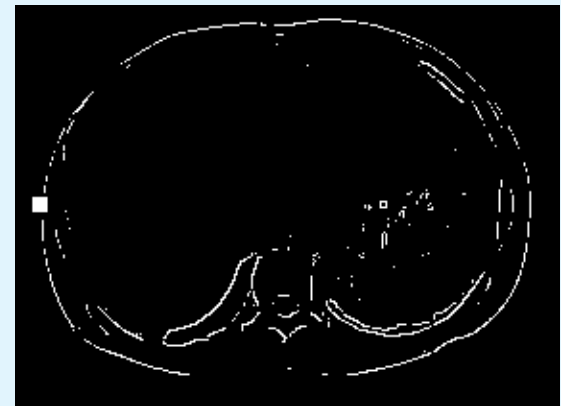

Figure 2(e): Output of Roberts edge detector.
In Figure 2(a) Original liver image is shown. It is possible to see the liver clearly. After applying the canny edge detector it is possible to see the liver boundary traced nicely by the gradient operator Canny and it is shown in Figure 2(b). The output images after applying the operator Sobel is shown in Figure 2(c). In this the boundaries are not very clear and dis continues. Even the shape of liver is not distinguishable clearly. In Figure 2(d) output image of Prewitt edge detector is presented. The boundaries are not clearly identified in this case also. Roberts operator output is given in Figure 2(e) which is not as good as that of the other operators.

Filtering is one efficient and effective method for enhancing the characteristics of an image. It removes the noise present in the image without sacrificing the 
characteristics of an image. In this work we have implemented many filters like hpf, lpf, mean median etc., and checked the output. It provided good results and enhanced the characteristics of the images considerably. While implementing soft thresholding techniques much better results are obtained. Fuzzy based filters provided not only excellent results but also natural like images for visual interpretation. Similarly wavelet based filters also provided excellent results. This study extensively likes to state that soft thresholding techniques based on fuzzy and wavelets filters are excellent as compared to the other classical image filters.

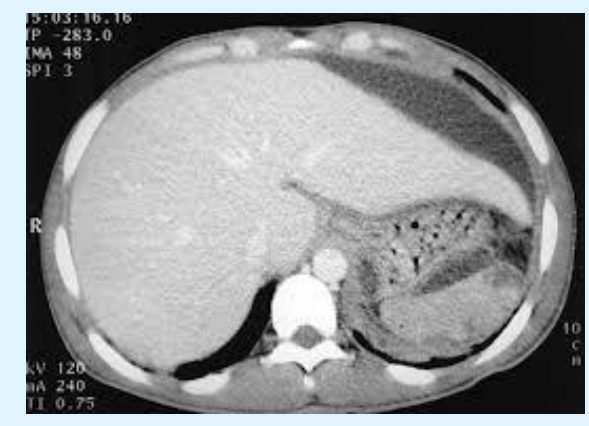

Figure 3(a): Original image.

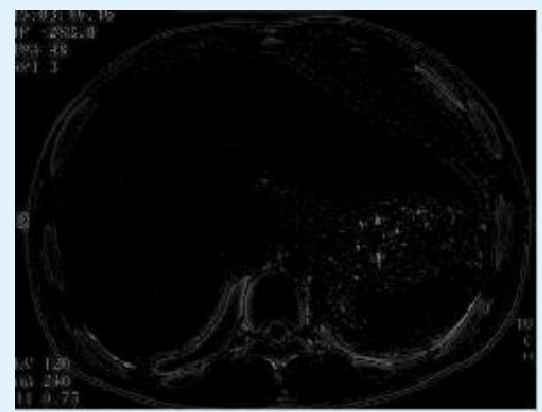

Figure 3(d): High Pass filter.

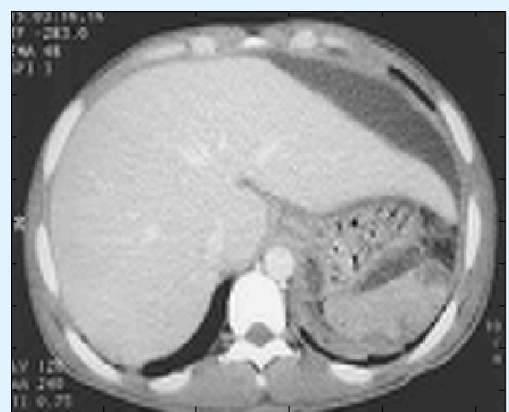

Figure 3(b): Fuzzy filter.

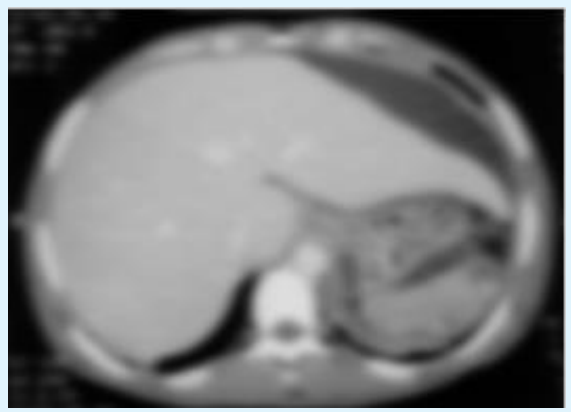

Figure 3(e): Low passes.

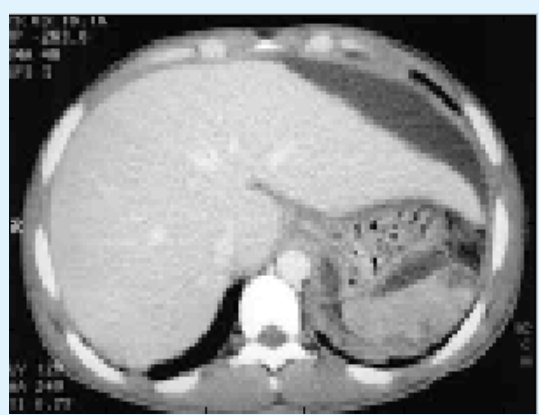

Figure 3(c): Wavelet filter.

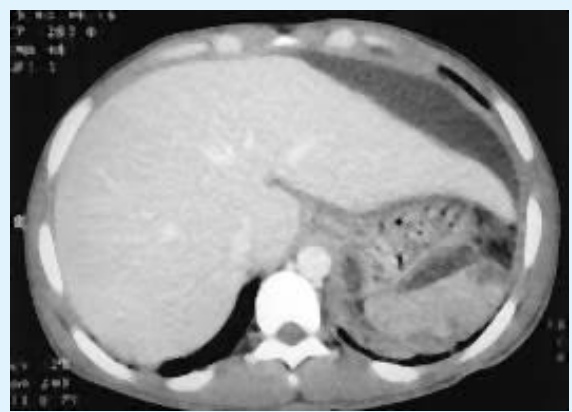

Figure 3(f): Mean.

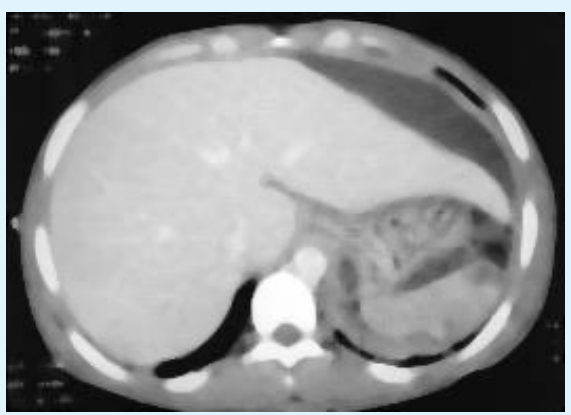

Figure 3(g): Median.

In Figure 3(a) original liver disease affected liver image is shown in Figures 3(a) \& 3(b) is the output image after applying the fuzzy filter. It is possible to observe that noise present in the image is completely removed and a smooth filtered output is obtained.
Figure 3(c) is the output image obtained after applying the wavelet filter. This is also found to be one of the best filters and the filtered image is a good smoothed image without noise. 
Among the classical filters high pass, low pass, mean and median filters are of considerable interest. In Figure 3(d) high pass filter output is presented. The image has turned black but the liver boundary is more prominent even in the presence of low contrast. Low pass filtered output is shown in Figure 3(e) and on visual inspection the output is not sufficiently good as compared to mean and median filters. Among these filters median filter is one of the best and hence used by image processing researchers long back. Mean filtered output is shown in Figure 3(f) and the Median filtered output is presented in Figure $3(\mathrm{~g})$

Hard thresholding is one of the methods of segmentation when object and background are clearly possible to visualize using grey levels. It is also considered as a process of partitioning pixels in an image into exclusive and exhaustive regions. In thresholding we have to identify a threshold $\mathrm{T}$ and then segment the image into two different regions for object and background. In real life situations it is not easy to identify a single threshold. The hard threshold output is shown in Figure 4(b). Otsu's method of segmentation (thresholding) is a hard thresholding technique. The output of which is shown in
Figure 4(c). Otu's method of segmentation based on thresholding maximizes the class separation of object and background. This method provides excellent results for a vide verity of natural images.

Fuzzy based thresholded output is shown in Figure 4(d). Zadeh LA in his paper exclusively stated that one of the areas of application of his fuzzy set theory is medical image analysis. Fuzzy thresholded image can be characterized by two membership functions, one for object and other for background after segmentation.

Wavelet based thresholding is an excellent method of segmentation. It can also provide high accuracy and segments the liver using global threshold. After enhancing the liver regions segmentation is performed to separate the image foreground and background. By segmenting an image also saves processing time. Segmentation can be done with global threshold or using multiple thresholds. Wavelet based techniques always provides excellent segmentation results with more than $90 \%$ accuracy as compared to all the other segmentation techniques. In Figure 4(e) wavelet based thresholding (segmentation) is implemented.

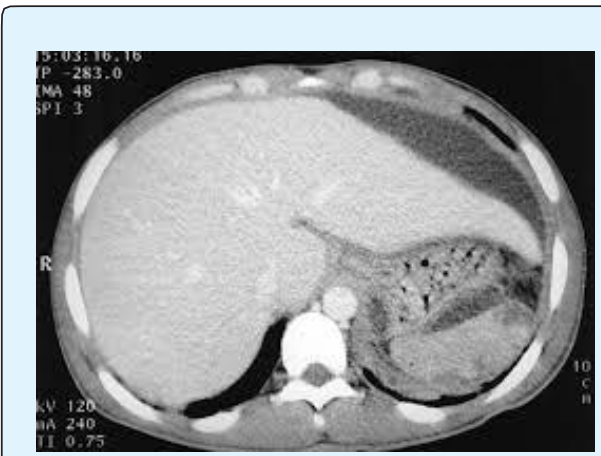

Figure 4(a): Original.

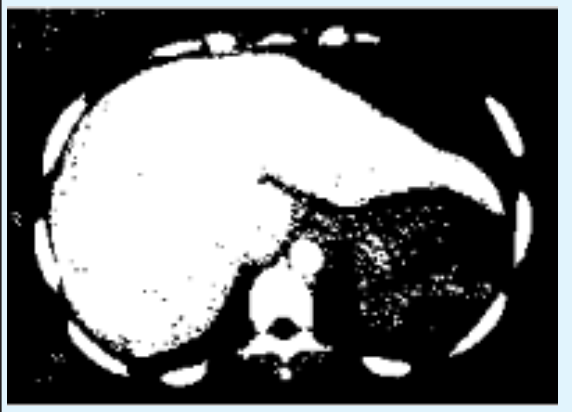

Figure 4(d): Fuzzy.

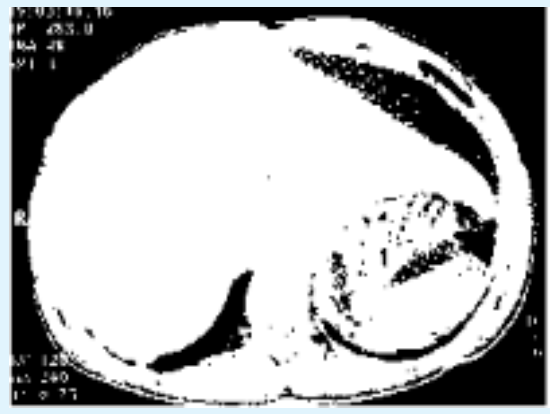

Figure 4(b): Thresholding.

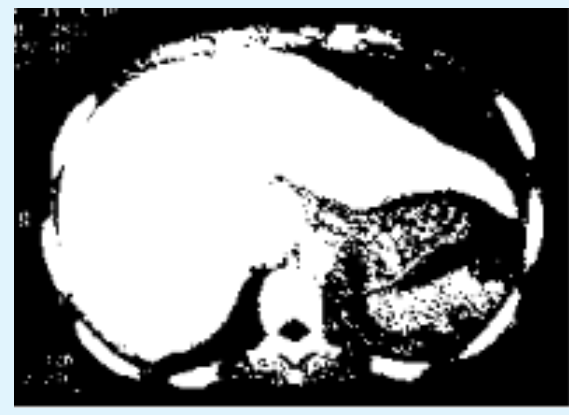

Figure 4 (e): Wavelet.

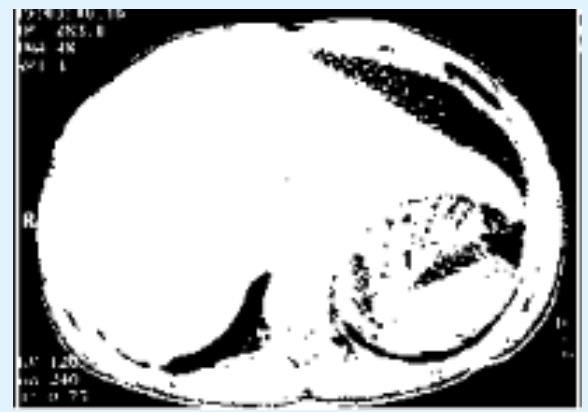

Figure 4(c): Otsu.

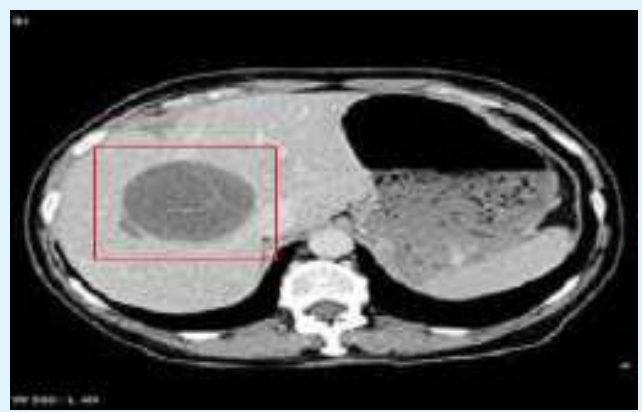

Figure 4(f): Original image. 


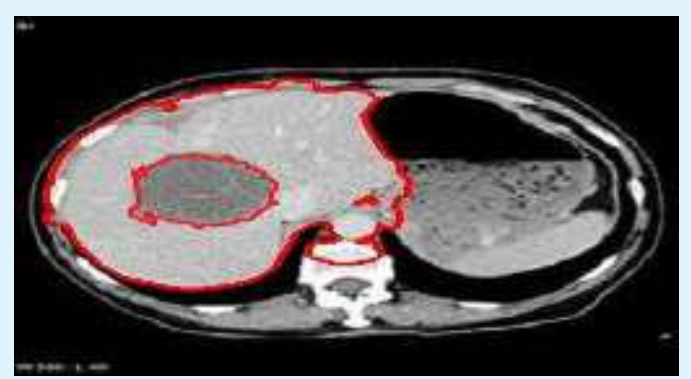

Figure 4(g): output of Level set method.

The level set methods are versatile, most efficient and accurate for a wide range of problems. This methods works be embedding propagate fronts as zero level set of high dimensional function. The equation of motion of this is like hammilton-Jacob equation. The critical value partial differential equation is solved using hyperbolic conservation laws. By using narrow band adaptive techniques the computational complexity can be reduced to some extent. This method can handle sharp corners and cusps. The main advantages of this method are increased accuracy and robust modeling.

In the area of liver mage analysis, scientists developed several techniques for segmenting liver tumors. Each one has its own merits and de-merits. The level set method of liver image processing has contributed several interesting results. In Figure 4(f) a liver tumor image is shown. Level set method is applied and the result is shown in Figure $4(\mathrm{~g})$. It is possible observe that the tumor boundary and liver boundary are clearly demarcated in this case. Using level set method it is possible to bring out clinical information. Further processing may provide more and more formation about the tumor.

Filtering techniques are extremely important while dealing with medical images. Hence wide verities of filters are used in medical image processing and related areas. The most commonly used conventional filters are the low pass filters, high pass filters, median filters mean filters etc. While processing with abdominal CT images it is observed that median filter performed well, out of all these. This is one of the old techniques but satisfactory for preliminary processing for advanced image processing more sophisticated filtering technique is necessary. Researchers put their effort in developing various techniques and as a result fuzzy filters and wavelet filters have been developed. While applying these filters to CT images of the liver very good filtered outputs are obtained. Hence these methods are found to be one of the most useful and effective method in image processing and pattern recognition.

Segmentation of images is very much required in the area of medical imaging for disease diagnosis and therapy. Segmentation of tumors and cysts are required in several cases for diagnosis and therapy. In liver image analysis tumor or cysts segmentation is possible and we will get more and more information about the tumor. This will definitely help in diagnosis and therapy.

We have applied the liver images to all the conventional segmentation techniques such as region growing, splitting and merging and thresholding. Thresholding techniques can be divided into two, hard thresholding and soft thresholding. Hard thresholding belong to a group of segmentation techniques modified by few researches like Otsu, Kapur, P K Sahoo and Kittler etc. Soft thresholding belongs to methods similar to Fuzzy thresholding, wavelet thresholding etc. we have implemented both in the case of abdominal images of liver CT and found that Fuzzy based thresholding and wavelet based thresholding provides excellent segmented output as compared to all the other segmentation methods.

\section{Texture Based Classification of CT Liver Images}

The summary of the proposed work on classification of liver tumors into benign and malignant are depicted in Figure 1 . The speciality of this work is that we have tried to obtain the optimum results by making use of the best methods used in each and every step. As an example for filtering we used fuzzy filters and wavelet filters and for segmentation fuzzy thresholding and wavelet thresholding are employed.

Texture characteristics can be used for the analysis of different types of images. These characteristics can also 
be used for identifying different regions in an image. This will assign the give texture region to known set of the texture classes. Texture analysis is considered as a challenging area of research due to its difficulty to analyze both natural and artificial textures using a single method. Texture based image analysis can be broadly classified into structural, statistical, model based and signal processing methods. All these methods use textures on single scale. In order to overcome this limitation multi resolution analysis can be used. This can easily be realized with the help of wavelet transforms.

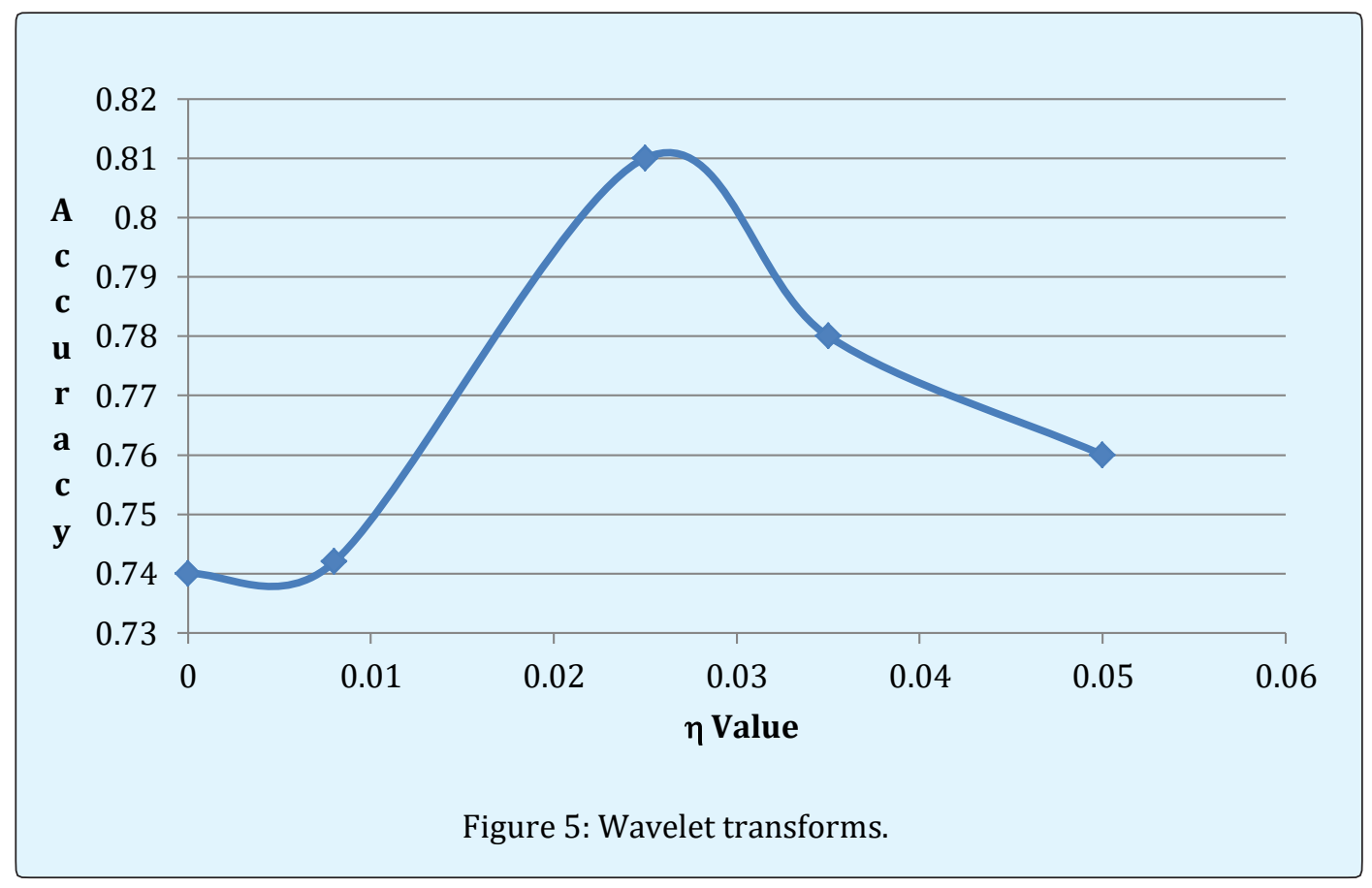

\section{Conclusions}

In this work various image processing operations are performed on liver CT images. This includes classical segmentation techniques followed by the latest methods which are available in the field of liver image analysis. Finally we compare these methods to check which the best options are in liver CT image analysis. Several input images and output images are experimented and the results are shown to the expert radiologist for their opinion and advice to examine whether any clinical information can be drawn from it. The images selected by them are further examined to find which methods are efficient in bringing clinical information without sacrificing image characteristics. Based on these studies we arrive at the following conclusions:

Among the edge based segmentation techniques level set methods out perform all the other methods. Earlier studies have also revealed that canny edge detector is the best edge detection technique. Sobel operator is also good but it not only enhances the unwanted edges but also smooth the image to some extent.
Among the conventional image filtering techniques such as LPF, HPF and Mean etc., Median filtering outperform all the other filters. It is observed that fuzzy filters and wavelet based filters provided the best results. Thresholding is one of the most simple and easy to implement segmentation technique without much computational complexity. These thresholding techniques commonly used by the image processing community have been implemented and the results are manually examined by the expert radiologist. Among the crisp thresholding techniques otsu's method provided best segmentation. However it is not good in providing internal details of the liver. Hence tumors or Cysts cannot be clearly identified. Also it changes the image completely with only one threshold.

Among the latest developments fuzzy thresholding and wavelet based thresholding provided the best results. It accomplishes the segmentation without changing the input image characterizes but brings the output with this clear image and possible to highlight clinical information. After applying these techniques excellent segmentation results are obtained than any other classical segmentation 
techniques. It is also observed that the times taken for performing these operations are relatively less due to less computational complexity.

\section{Acknowledgement}

The authors like to thank Dr. K. Ramachandran, Professor in imageology, Regional Cancer Center, Medical College campus, Thiruvananthapuram. Kerala, India for providing the CT images of liver.

\section{References}

1. Pal NR, Pal SK (1993) A review of image segmentation techniques. Pattern Recognition 26(9): 1277-1294.

2. Kumar P, Bhalerao BS (2014) Detection of tumor in liver using image segmentation and registration technique. IOSR Journal of Electronics and Communication Engineering 9(2): 110-115.

3. Singh KK, Singh A (2010) A study of image segmentation algorithms for different types of images. International journal of computer science 7(5): 1-4.

4. Ramanjot Kaur, Lakh winder kaur, Savita Gupta (2011) Enhanced K-means clustering algorithm for liver image segmentation to extract cyst region. International journal of computer applications pp: 59-66

5. Priyadarshini S, Selvathi D (2012) Survey on segmentation of liver from CT images. IEEE International conference on Advanced Communication control and computing technologies, pp: 234-238.

6. Marius George Linguraru, William J Richbourg, Jianfei Liu, Jeremy M Watt, Vivek Pamulapat, et al. (2012) Tumor burden analysis on computer tomography by automated liver and tumor segmentation. IEEE transactions on medical imaging 31(10): 1965-1976.

7. Bezdek JC, Pal SK (1992) Fuzzy models for pattern Recognition. IEEE press, New York.

8. Zadeh LA (1965) Fuzzy sets, Information and control 8(3): 338-353.

9. Krishnapuram R, Frigui H, Nasraoui O (1995) Fuzzy and probabilistic shell clustering algorithms and their application to boundary detection and surface
approximation-Part-1 \& Part-11, IEEE Transactions on Fuzzy systems 3(1): 29-60.

10. Harralick RM, Sharpiro L (1992) Computer and Robot vision. Addison Wesley, USA I-II.

11. Jawahar CV, Roy AK (1996) Incorporation of grey level imprecision in representation and processing of digital images, pattern Recognition letters 17(5): 541546.

12. Bezdek JC (1981) Pattern Recognition with fuzzy objective function algorithms, Plenum Press, New York.

13. Maruthy CA, Pal SK (1990) Fuzzy thresholding: Mathemmatical framework, bound functions and weighted moving average technique pattern Recognition letters 11(3): 197-206.

14. Pal SK, Rosenfield A (1990) Image enhancement and thresholding by optimization of Fuzzy compactness. Pattern Recognition letters 7(2): 77-86

15. Ridler T, Calvard S (1978) Picture thresholding using an interactive selection method. JEEE Trans, Systems, Man \& Cybermatics 8(8): 630-632.

16. Huang L, Wang MJ (1995) Image thresholding by minimizing the measure of fuzziness. Pattern Recoguition 28(1): 44-51.

17. Fung PW, Ly KK, Attijiouzel K (1998) Automatic segmentation of biomedical images. Proce: IEEE conf, Acoustics, speech \& signal processing, pp: 882-885.

18. Rosenfield A, Hunnel R, Suker S (1976) Sene labeling by relaxation operation. IEEE Transactions on systems, Man \& Cybernatics 6(6): 420-433.

19. Mejer F, Beucher S (1990) Morphological segmentation. Journal of visual communication and image representation 1(1): 21-46.

20. Harsen MW, Higgins WE (1994) Watershed drivers Relaxation labeling for image segmentation. IEEE Int Conf on Image processing 3: 460-464

21. Dolaboljian C, Jalal Huertasleyva MFE (2002) Classical low pass filter and real time wavelet based denoising technique implemented on a DSP. A comparison study of low pass filter and real time Wavelet based denoizing 20(2): 135-140. 
22. Aldroubi A, Unser M (1996) Wavelets in medicine and biology. CRC Pars Boca Raton FL, USA.

23. Rupinder Pal Kauretal (2013) Survey of denoising methods using filters and last wavelet transforms. SARCSSE 3(2): 133-136.

24. Gonzales RC (2006) The field methods in remote sensing, $2^{\text {nd }}(E d n$.$) , Rawart Publications.$

25. Daubechies I, Sweldms W (1998) Factoring wavelet transforms into lighting steps. Fourier analysis applications 4(3): 247-269.

26. Vile DVD, Nachtegat M, Van der Wakeu, Kerre EE, Philips W, et al. (2003) Noise reduction by fuzzy image filtering. IEEE Transactions on Fuzzy systems 11(4): 429-436.

27. Subba Reddy PV (2017) Fuzzy logic based on belief and disbelief membership functions. Fuzzy information engineering 9(4): 405-422.

28. Ping R (1990) Fuzzy sets and representation of incomplete knowledge. Fuzzy sets and systems 36(1): 91-96.

29. Zadeh LA (1965) Fuzzy sets. Information \& Controls 8(3): 336-353.

30. Zedeh LA (2006) Generalized theory of uncertainty principal concepts and ideas. Computational Statistics \& Data Analysis 51(1): 15-46.

31. Taheri S, Ong SH (2010) Level set segmentation of brain tumors using threshold based speed function. Image and vision computing 28(1): 26-37.

32. Wan M, Guohua Gu, Jianhong Sun, Weixian Qian, Kan Ren, et al. (2018) A level set method for infrared image segmentation using Global and Local Information. MDPI 10(7): 1039.

33. Chodhry MS, Kapur R (2018) A novel energy based level set method for medical image segmentation. Cogent engineering 5(1): 1475032.

34. Maolood IY, Abdulridha YE, Al-Salahi, Songfeug Lu (2018) Thresholding for medical image segmentation for cancer using fuzzy entropy with level set algorithm. Open med (wars) 13(1): 374-383.

35. Wang D (2018) Hybrid fitting energy based fast level set model for image segmentation solving by algebraic multi grid and sparse field method. IET image processing 12(4): 539-545.

36. Otsu N (1977) A threshold selection method from grey level histograms. IEEE Transactions on Systems, Man and cybernetics 9(1): 62-66.

37. Kapur JN, Sahoo PK, Wong AKC (1985) A new method for grey level picture thresholding using the entropy of the histogram. Computer vision graphics and image process 29(3): 273-285

38. Sahoo PK, Soltani S, Wone AKC (1988) A Survey of thresholding techniques. Computer vision graphics and image processing 41(2): 233-260.

39. Senthil Kumarn N, Vaithegi S (2016) Image segmentation is using thresholding technique for medical images. International national journal of computer science 6(1): 1-30.

40. Sujan M, Alarm N, Abdhula H, Jairul M (2016) A Segmentation based Automated System for Brain Tumor Detection. International journal of computer applications 153(10): 41-49.

41. Shanti S, Vamsidhar E (2017) Implementation of Fuzzy thresholding for segmentation of images. International journal of computer applications 180(2): 46-51.

42. Hajar Alharbi, Paul Kwan, Sajeev ASM (2012) A Comparative study of Fuzzy thresholding techniques for mas detection in digital mammography, pp: 330334

43. Rajesh Joshi, Satish Kumar (2018) An expositional Jensen fuzzy divergence measure with applications in multiplied attribute decision making. Mathematical problems in Engineering 2018: 9.

44. Aruna Kumar SB, Harish BS (2017) A modified intutionistic fuzzy clustering algorithm for medical image segmentation. Journal of intelligence systems $27(4)$.

45. Umit L, Ahamed L (2017) Brain tumor segmentation based on a new threshold approach. Procedia computer science 120: 580-587.

46. Rakesh Kumar, Saini BS (2012) Improved image denoising technique using neighboring wavelet coefficient of optimal wavelet with adapting thresholding. International journal computer theory and Engineering 4(3): 1-6. 
47. Fathi A, Ahamed Raza NN (2012) Efficient image denoising method based on new adapting wavelet packet thresholding function. IEEE transactions on image processing 21(9): 3981-3990.

48. Virendrakumar, Ajyakumar (2013) Simulative analysis of image de noising using wavelet thresholding techniques. International journal of advanced research in computer engineering and technology 2(5): 1-6.

49. Donoho DL, Johnstone IM (1995) De noising by soft thresholding. IEEE transaction on information theory 41(3): 613-627.
50. Jabamalar Leavline E, Sutha S, Azir Antoney, Ganga Singh D (2013) A comprehensive study on wavelet based shrinkage methods for de noising natural images. WSEAS Transaction on signal processing 9(4): 1-13.

51. Jabamalar Leavline E, Sutha S, Azir Antoney, Ganga Singh D (2011) Wavelet domain shrinkage methods for noise removal in images: a compendium. International journal of computer application 33(10): $1-5$.

52. Sun, Wee (1983) Neighboring grey level dependent method. Computer vision graphics and image processing 23(3): 342-352. 